\title{
Polycystic Ovarian Syndrome: Role of Genes and Genetics
}

\author{
Supriya Roy and Pranati Srivastava* \\ Amity Institute of Pharmacy, Amity University Uttar Pradesh, Lucknow, Uttar Pradesh, India
}

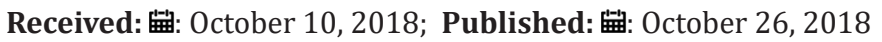

*Corresponding author: Ms. Pranati Srivastava, Amity Institute of Pharmacy, Amity University Uttar Pradesh, Lucknow, India

\begin{abstract}
The most common systemic and an inflammatory endocrine-pathology that affects about 5-20\% of women of reproductive age is polycystic ovary syndrome (PCOS). PCOS, also discussed as polycystic ovaries (PCO) or polycystic ovarian disease (PCOD), is a heterogeneous disorder having multifactorial etiology involving genetics, environmental factors as well as fetal and childhood exposures. The pathophysiology is characterized by the hallmark features of hyperandrogenism and insulin resistance. Insulin resistance can also aggravate hyperandrogenism. The typical triad of this syndrome-obesity, chronic anovulation and hirsutism are mainly the result of hyperandrogenism. Other symptoms may include pain in the pelvic region, male pattern alopecia, irregular periods and acne and can ultimately lead to infertility. Transformed expression of fundamental enzymes involved in the steroidogenic pathway may contribute to amplify ovarian steroidogenesis patterns. Candidate gene involved are under persistent investigation to demarcate the association of PCOS development with polymorphic genes, which encode for various enzymes involved in steroidogenesis or modify the levels and activity of intrinsic androgens. In the current review, the effect of androgen related genetic polymorphisms in governing genetic predisposition to PCOS and its linked metabolic and reproductive traits is summarized.

Abbreviations: Polycystic Ovary Syndrome (PCOS), Polycystic Ovarian Disease (PCOD), Polycystic Ovaries (PCO), Insulin gene (INS), Insulin Receptor Gene (INSR), Insulin Receptor Substrate Genes (IRSs), Calpain-10 Gene (CAPN10), Serum Sex Hormone-Binding Globulin (SHBG), Variable Tandem Repeats (VNTR)
\end{abstract}

\section{Introduction}

PCOS is the most common hormonal abnormality and the common cause of infertility that affects the reproductive-age women. About 5 to 20 percent of females with metabolic syndrome such as diabetes, obesity and cardiovascular disease [1] are estimated to be associated with an increased risk of PCOS. Although the symptoms of PCOS can vary, the syndrome is characterized by the presence of hallmarks symptoms such as chronic anovulation, polycystic ovaries, hyperandrogenism and irregular menstruation [2]. PCOS is a heterogeneous disorder that involves altered expression of chronic anovulation and hyperandrogenism due to defective or faulty cell specific control mechanisms [3]. Interaction of multiple intrinsic genes with external environmental factors is supposed to be the one of the major contributing factor. Although full investigation and understanding of the genes and the contribution of the various environmental factors involved in the etiology of the syndrome is still unelaborated. Segmentation of the genetic basis of PCOS is presently an area of intensive exploration, [4] with more than 70 genes that have been evaluated for their impact on the etiology of PCOS. Of all genes involved in the development of insulin resistance plays a central key element contributing to PCOS [5]. Insulin controls metabolic homeostasis and adds to ovarian steroidogenesis that is further aggravated by obesity. Thus, association have been demonstrated between gene involved in insulin secretion and action and PCOS susceptibility.

\section{Various Genes Involved in Syndrome Inheritance}
a) CYP11A
b) CYP17A1
c) CYP19
d) CYP21

e) Serum Sex Hormone Binding Globulin (SHBG)

f) Leptin

g) Adiponectin

h) Insulin gene (INS)

i) Insulin receptor gene (INSR)

j) Insulin receptor substrate genes (IRSs)

k) Calpain-10 gene (CAPN10)

A gene CYP11A encodes for cytochrome P450 enzyme. The enzyme is responsible for the catalyzing the first step of 
steroidogenesis i.e. conversion of cholesterol into progesterone [6-10]. Several investigational studies confirmed the confirmation for the association of CYP11a with the pathogenesis of PCOS. The expression of CYP17 is also dysregulated at the level of mRNA stability in PCOS theca cells. CYP19 is responsible for the conversion of androgen to estrogen [11]. Enzyme complex aromatase is encoded by CYP19 and deficiency of aromatase in PCOS follicles leads to excess androgen production and thereby improper follicle development. 21-Hydroxylase enzyme encoded by CYP21 converts 17-hydroxyprogesterone into 11-deoxycortisol which is another main step in ovarian or adrenal steroidogenesis. An increased level of serum 17-hydroxyprogesterone have been investigated in women with PCOS which the result of mutation of CYP21 gene may be [12]. A gene (AR) located at Xq11-12 encodes for the androgen receptor. Various studies established that an increased level of androgen receptor activity associated with decreased number of CAG [13-15] repeats could explain hyperandrogenism and symptoms associated with it in PCOS women. Serum Sex Hormone-Binding Globulin (SHBG) [16] produced by hepatocytes is a homodimer glycoprotein that is encoded by 4-kb gene. Low levels of SHBG are estimated in patients with hyperandrogenism and PCOS.

The adipocytokines genes such as adiponectin and leptin have been explored as one of the major candidate genes involved in the pathogenesis of PCOS. Leptin help to regulate energy balance. Higher percentage of body fat higher the leptin is released by adipocytes [17-19]. Elevated levels of leptin have been observed in women with PCOS. Leptin not only aids in the production of steroid hormones in the ovary but also stimulate reproductive system that contribute to ovarian overproduction of androgens and hyperandrogenemia [20]. Both insulin and obesity contribute to the elevated leptin levels because of increased leptin mRMA in adipocytes. Research studies demonstrated that free leptin index is elevated, and leptin receptor are decreased in case of PCOS. It can also be possible that leptin levels are elevated secondary to hyperinsulinemia as consequence of insulin-stimulated synthesis of leptin [21] Both leptin and insulin support ovarian overproduction of androgens and lead to hyperandrogenism and express a positive relationship between leptin and insulin in PCOS. Polymorphism of adiponectin gene T45G and G276T are found to be allied with insulin resistance and obesity.

But results depicting the relation between the gene polymorphisms and serum adiponectin level are still conflicting. However, it has been observed that serum adiponectin levels are lowered by the carriers of G allele in PCOS. Polymorphism of variable tandem repeats (VNTR) in the insulin gene (located at the 5 regulatory region of INS) has been found accountable for directing the rate of transcription of insulin. It showed an extensive outcome on the insulin resistance and hyperinsulinemia in PCOS. The male-pattern baldness has also been observed to be associated with INS VNTR polymorphisms. A heterotetrametric glycoprotein, insulin receptor is encoded by the gene INSR positioned at the chromosome 19. An extensive region of the chromosome so called 19p13.2 was explored to be associated with D19S884. Data suggest that the insulin resistance in PCOS women can be the outcome of mutations of INSR. Insulin resistance has also found to be allied with polymorphisms of insulin receptor substrate genes such as IRS1 and IRS2.

The polymorphic influence of Gly972Arg IRS-1 and of Gly1057Asp IRS-2 [22] was scrutinized in non-diabetic women with PCOS and the results revealed that the Gly972Arg IRS-1 was more ubiquitous in patients resistant to insulin than non-insulin resistant patients. Insulin secretion and physiology is governed by a cysteine protease called calpain-10. Calpain-10 is encoded by gene CAPN10. Effect of gene variation in the CAPN10 may have part in the pathogenesis of PCOS. 112/121 haplotype of calpain gene is supposed to possess higher risk for the development of the PCOS.

\section{Conclusion}

PCOS is a common endocrine ailment that has a robust genetic part and is characterized by symptoms like polycystic ovaries, hyperandrogenemia, and menstrual irregularity. PCOS is a multifactorial and a complex reproductive pathology wherein a diversity of influencing genes interacts with various lifestyles and environmental factors to produce etiology. More than 70 candidate genes have been assessed for an underlying role in PCOS during the past decade. However, because of underpowered studies and complex genetic and phenotypic heterogeneity, the results of many of these studies remain inconclusive. Studies demonstrated that the PCOS has a heritable nature that has led to the enormous population studies endeavoring to determine the role of various genes that influence PCOS using the candidate gene approach. The appropriate tools such as correctly sized PCOS cohorts and genetic reagents are now considered to carry out extensive studies to analyze the genetic basis of PCOS. This may lead promising and productive effects in coming 10 years for the proper management and treatment of the syndrome.

\section{References}

1. Mykhalchenko K, Lizneva D, Trofimova T, Walker W, Suturina L, Diamond MP et al. (2017) Genetics of polycystic ovary syndrome. Expert Rev Mol Diagn 17(7): 723-733.

2. Azziz R, Carmina E, Chen Z, Dunaif A, Laven JS, Legro RS, et al. (2016) Polycystic ovary syndrome. Nat Rev Dis Primers 2: 16057.

3. Lizneva D, Suturina L, Walker W, Brakta S, Gavrilova Jordan L, Azziz R et al. (2016) Criteria, prevalence, and phenotypes of polycystic ovary syndrome. Fertil Steril 106(1): 6-15.

4. Sheikhha Mohammad Hasan, Kalantar Seyed Mehdi, Ghasemi Nasrin (2007) Genetics of polycystic ovary syndrome. Iran J Reprod Med Winter 5(1): 1-5.

5. Gunning MN, Fauser BCJM (2017) Are women with polycystic ovary syndrome at increased cardiovascular disease risk later in life? Climacteric 20(3): 222-227.

6. Hoeger K (2001) Obesity and weight loss in polycystic ovary syndrome. Obstet Gynecol Clin North Am 28(1): 85-97.

7. Maliqueo M, Lara HE, Sanchez F, Echiburu B, Crisosto N, et al. (2013) Placental steroidogenesis in pregnant women with polycystic ovary syndrome. Eur J Obstet Gynecol Reprod Biol 166(2): 151-155.

8. Urbanek M (2013) The genetics of the polycystic ovary syndrome. Nat Clin Pract Endocrinol Metab 373(1-2): 29-38. 
9. Crisosto N, Echiburu B, Maliqueo M, Perez V, Ladron de G, et al. (2012) Improvement of hyperandrogenism and hyperinsulinemia during pregnancy in women with polycystic ovary syndrome: possible effect in the ovarian follicular mass of their daughters. Fertile Sterile 97(1): 218-224.

10. Azziz R, Dumesic DA, Goodarzi MO (2011) Polycystic ovary syndrome: an ancient disorder. Fertil Steril 95(5): 1544-1548.

11. Homburg R, Ray A, Bhide P, Gudi A, Shah A, et al. (2013) The relationship of serum anti-Mullerian hormone with polycystic ovarian morphology and polycystic ovary syndrome: a prospective cohort study. Hum Reprod 28(4): 1077-1083.

12. (2014) A cross-sectional study of polycystic ovarian syndrome among adolescent and young girls in Mumbai, India. Indian J Endocrinol Metab 18(3): 317-324.

13. Xie GB, Xu P, Che YN, Xia YJ, Cao YX, et al. (2013) Microsatellite polymorphism in the fibrillin 3 gene and susceptibility to PCOS: a casecontrol study and meta-analysis. Reprod Biomed Online 26(2): 168-174.

14. Goodarzi MO, Azziz R (2006) Diagnosis, epidemiology, and genetics of the polycystic ovary syndrome. Best Pract Res Clin Endocrinol Metab 20(2): 193-205.

15. Urbanek M, Legro RS, Driscoll DA, Azziz R, Ehrmann DA, et al. (1999) Thirty-seven candidate genes for polycystic ovary syndrome: strongest

ISSN: 2574-1241

DOI: $10.26717 / B J S T R .2018 .10 .001951$

Ms. Pranati Srivastava. Biomed J Sci \& Tech Res

(C) This work is licensed under Creative Commons Attribution 4.0 License

Submission Link: https://biomedres.us/submit-manuscript.php evidence for linkage is with follistatin. Proc Natl Acad Sci U S A 96: 85738578.

16. Villuendas G, Escobar Morreale HF (2003) Association between the D19S884 marker at the insulin receptor gene locus and polycystic ovary syndrome. Fertil Steril 79(1): 219-220.

17. Murri M, Luque Ramirez M, Insenser M (2013) Circulating markers of oxidative stress and polycystic ovary syndrome (PCOS): a systematic review and meta-analysis. Hum Reprod Update 19(3): 268-88.

18. Jordan CD, Bohling SD, Charbonneau NL, Sakai LY (2010) Fibrillins in adult human ovary and polycystic ovary syndrome: is fibrillin-3 affected in PCOS? J Histochem Cytochem 58(10): 903-915.

19. Ferk P, Perme MP, Gersak K (2008) Insulin gene polymorphism in women with polycystic ovary syndrome. J Int Med Res 36(6): 1180-1187.

20. Gorsic LK, Kosova G, Werstein B, Sisk R, Legro RS et al. (2017) Pathogenic Anti-Müllerian Hormone Variants in Polycystic Ovary Syndrome. J Clin Endocrinol Metab 102(8): 2862-2872.

21. Xu Y, Wei Z, Zhang Z, Xing Q, Hu P et al. (2009) No association of the insulin gene VNTR polymorphism with polycystic ovary syndrome in a Han Chinese population. Reprod Biol Endocrinol 7: 141.

22. Lee EJ, Oh B, Lee JY, Kimm K (2008) A novel single nucleotide polymorphism of INSR gene for polycystic ovary syndrome. Fertile Sterile 89(5): 1213-1220.

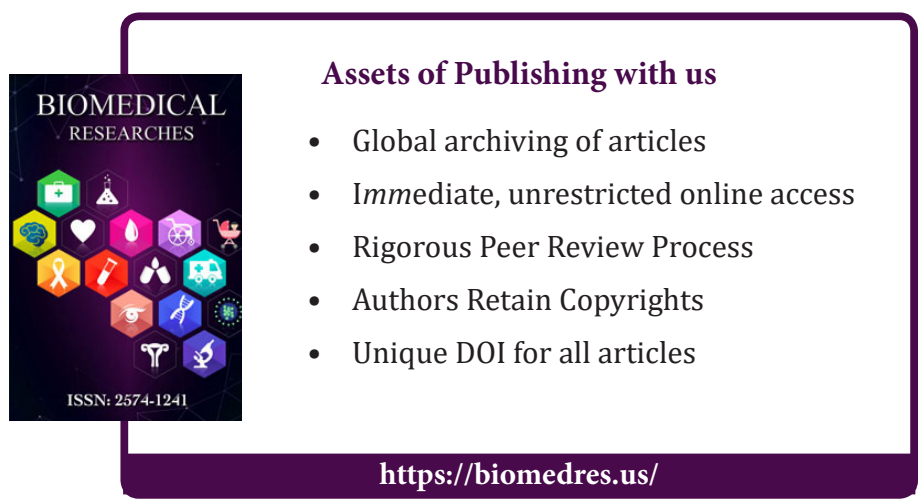

